\title{
Molecular epidemiological and virological study of dengue virus infections in Guangzhou, China, during 2001-2010
}

Liyun Jiang, Xinwei Wü, Yejian Wu, Zhijun Bai, Qinglong Jing, Lei Luo, Zhiqiang Dong, Zhicong Yang, Yang Xu, Yimin Cao, Biao Di, Yulin Wang and Ming Wang

\begin{abstract}
Background: Dengue virus (DENV) infection is the most prevalent arthropod-borne viral infection in tropical and subtropical regions worldwide. Guangzhou has the ideal environment for DENV transmission and DENV epidemics have been reported in this region for more than 30 years.

Methods: Information for DENV infection cases in Guangzhou from 2001 to 2010 were collected and analyzed. The DENV strains were cultured and isolated from patients' sera. Viral RNA was extracted from cell culture supernatants. cDNA was synthesized by reverse transcription PCR. Phylogenetic trees of four DENV serotypes were constructed respectively.
\end{abstract}

Results: In total, 2478 DENV infection cases were reported; 2143 of these (86.43\%) occurred during 3 months of the year: August, September and October. Of these, 2398 were local cases (96.77\%) and 80 were imported cases (3.23\%). Among the imported cases, 69 (86.25\%) were from Southeast Asian countries. From the 90 isolated strains, $66.67 \%, 3.33 \%, 14.44 \%$, and $15.56 \%$ belonged to DENV serotypes $1,2,3$, and 4 , respectively. DENV-1 was predominant in most of the years, including during 2 outbreaks in 2002 and 2006; however, none of the strains or genotypes identified in this study were found to be predominant. Interestingly, DENV strains from different years had different origins. Moreover, the strains from each year belonged to different serotypes and/or genotypes.

Conclusions: Southeast Asia countries were found to be the possible source of DENV in Guangzhou. These findings suggest that there is increasing diversity in DENV strains in Guangzhou, which could increase the risk of DENV outbreaks in the near future.

Keywords: Dengue virus, Infection, Molecular biology, Phylogenetic analysis, South China

\section{Background}

Dengue virus (DENV) is a member of the genus Flavivirus, family Flaviviridae. It is an enveloped virus with an 11-kb positive sense single-stranded RNA genome. The genome encodes a single open reading frame and can be translated into 3 structural proteins, that is, the core $(\mathrm{C})$, premembrane/membrane (prM/M), and envelope (E) proteins, and 7 non-structural proteins, that is, NS1, NS2a, NS2b, NS3, NS4a, NS4b, and NS5. DENV can be divided into 4 serotypes and several genotypes according to the sequence of the $\mathrm{E}$ gene. DENV infection can cause differential

\footnotetext{
* Correspondence: tom.wu@tom.com

Guangzhou Center for Disease Control and Prevention, 1 Qide Road, Guangzhou, Guangdong 510440, China
}

(c) 2013 Jiang et al.; licensee BioMed Central Ltd. This is an Open Access article distributed under the terms of the Creative Commons Attribution License (http://creativecommons.org/licenses/by/2.0), which permits unrestricted use, distribution, and reproduction in any medium, provided the original work is properly cited. gue fever (DF) to lethal complications like dengue hemorrhagic fever (DHF) and dengue shock syndrome (DSS) [1,2]. Infection with any of the 4 serotypes can cause extremely severe manifestations. Moreover, natural infection with any of the serotypes can only provide long-term homotypic immunity, which leads to a higher risk for DHF/DSS during secondary infections with a heterogenous serotype [3,4].

DENV infection has been the most prevalent arthropodborne viral infection in tropical and subtropical regions worldwide. There are 2 competent vectors of DENV, Aedes aegypti and Ae. albopictus. According to World Health Organization (WHO) estimates, over 100 countries and 
approximately $40 \%$ of the world's population (nearly 2.5 billion people) are threatened by DENV $[5,6]$. Currently, approximately 100 million DENV infections occur worldwide every year, and the WHO has classified dengue as a major international public health concern. Globalization has also increased the spread of viruses and mosquito vectors; hence, epidemics and outbreaks have occurred with increased frequency in recent years $[7,8]$. The diversity of the virus is also increasing since multiple DENV serotypes can co-circulate in the same location within a short time. Evolution, dispersal, and replacement of serotypes and genotypes could also take place during a continuous period under selection pressure [9-13].

Since the first documented DENV infection in Foshan in 1978, DENV has spread in China for more than 30 years, mostly in the south-east coast of China in regions such as the Guangdong, Shanghai, Jiangsu, Zhejiang, Hainan, Guangxi, and Fujian provinces [14]. Guangzhou, which is one of the biggest cities in South China, has a high incidence of DENV infections and in recent decades, DENV infection cases have been recorded in Guangzhou almost every year. The cases include both imported and indigenous cases, and all 4 DENV serotypes have been involved. In this study, epidemiological information pertaining to DENV infection cases that occurred between 2001 and 2010 in Guangzhou was reviewed. DENV strains from DF patients were isolated, and phylogenetic analysis was performed based on the E gene sequences. By combining epidemiological and virus serotype data from DENV cases occurring in Guangzhou between 2001 and 2010, we provide further insights into the transmission patterns of DENV in Guangzhou.

\section{Results}

\section{Epidemiological characteristics of DENV in Guangzhou} from 2001 to 2010

Information for all DENV infection cases was collected by the Guangzhou Center for Disease Control and Prevention (Guangzhou CDC). In total, 2478 DENV infection cases were reported; both laboratory and clinically confirmed cases were covered. All the infected patients had DF symptoms; however, no deaths or DHF/DSS cases were found. DENV infections were detected every year in Guangzhou from 2001 to 2010. Figure 1 shows the monthly distribution of reported DENV infection cases; $86.43 \%$ (2143 cases) of them occurred during 3 months of the year: August, September, and October.

There were 2398 local cases (96.77\%) and 80 imported cases (3.23\%) (Table 1). Among the imported cases, 69 (86.25\%) came from Southeast Asian countries, including Cambodia, Indonesia, Malaysia, Myanmar, Philippines, Singapore, Thailand, Vietnam, Bangladesh, and India. In addition, 5 (6.25\%) were imported from Africa (Niger, Senegal, South Africa, Tanzania, and Sudan), 2

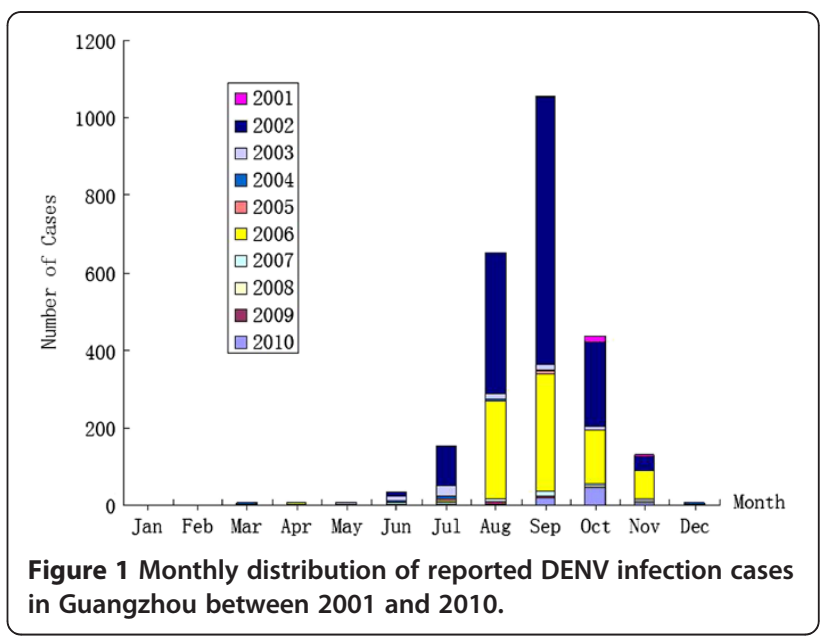

(2.50\%) from the Middle East (Saudi Arabia and Yemen), and 1 (1.25\%) from America (Dominican Republic). The original source of 3 (3.75\%) imported cases could not be identified. The imported cases outnumbered the local cases in 2005, 2008, and 2009. Two major outbreaks occurred in 2002 and 2006.

\section{DENV strains in Guangzhou from 2001 to 2010}

We isolated 90 strains from the serum samples of patients by using C6/36 cell line. The full-length DENV E gene sequences were then analyzed and compared. The sequences from 76 strains were deposited in GenBank with the following accession numbers: JN009085-99, HM466962-8, JQ002658, and JQ277834-86. The remaining 14 sequences were found to be identical sequences and were therefore not deposited. All the sequences were named using 2 numbers, one indicating the year and the other indicating the sample number. For example, 07-5668 means the strain was isolated in 2007 and the original sample number is 5668 . The reference strain sequences were downloaded from Genbank and named with the serotype, country, separation year, and accession number. In total, 10 of the isolated strains came from imported cases (Table 2), whilst the remaining 80 strains originated locally.

Among the 90 strains, 66.67\%, 3.33\%, 14.44\%, and $15.56 \%$ belonged to DENV serotypes 1,2 , 3, and 4, respectively. Table 3 shows the different serotypes for each year. Although DENV-1 was predominant in most years of the decade, DENV-2, DENV-3, and DENV-4 were occasionally isolated in 2005 and 2009. In 2010, all 4 DENV serotypes appeared were detected.

\section{Phylogenetic analysis of DENV-1}

Sequences of the E gene from the 60 DENV-1 isolates were aligned using ClustalW [15] and compared with 38 reference sequences in GenBank. Figure 2 shows the phylogenetic tree of the sequences using the maximum likelihood analysis and indicates that DENV-1 in Guangzhou falls 
Table 1 Summary of reported DENV infection cases in Guangzhou in 2001-2010

\begin{tabular}{|c|c|c|c|c|c|c|c|c|c|c|c|}
\hline Year & 2001 & 2002 & 2003 & 2004 & 2005 & 2006 & 2007 & 2008 & 2009 & 2010 & Total cases \\
\hline Total cases & 19 & 1423 & 78 & 21 & 12 & 779 & 35 & 12 & 18 & 81 & 2478 \\
\hline Local cases & 18 & 1419 & 76 & 15 & 1 & 771 & 22 & 4 & 6 & 66 & 2398 \\
\hline Imported cases & 1 & 4 & 2 & 6 & 11 & 8 & 13 & 8 & 12 & 15 & 80 \\
\hline \multicolumn{12}{|l|}{ Country of origin } \\
\hline Bangladesh & 0 & 0 & 0 & 0 & 1 & 0 & 1 & 0 & 0 & 0 & 2 \\
\hline Cambodia & 0 & 0 & 0 & 2 & 1 & 4 & 4 & 0 & 0 & 1 & 12 \\
\hline Dominican Republic & 0 & 0 & 0 & 0 & 0 & 0 & 1 & 0 & 0 & 0 & 1 \\
\hline India & 0 & 0 & 0 & 1 & 2 & 0 & 0 & 0 & 0 & 1 & 4 \\
\hline Indonesia & 1 & 1 & 0 & 2 & 2 & 2 & 1 & 1 & 2 & 2 & 14 \\
\hline Malaysia & 0 & 0 & 0 & 0 & 1 & 0 & 2 & 2 & 1 & 1 & 7 \\
\hline Myanmar & 0 & 0 & 0 & 0 & 0 & 0 & 1 & 0 & 0 & 0 & 1 \\
\hline Nigeria & 0 & 0 & 0 & 0 & 0 & 0 & 0 & 0 & 0 & 1 & 1 \\
\hline Philippines & 0 & 0 & 0 & 0 & 0 & 0 & 0 & 0 & 2 & 0 & 2 \\
\hline Saudi Arabia & 0 & 0 & 0 & 0 & 0 & 0 & 0 & 0 & 1 & 0 & 1 \\
\hline Senegal & 0 & 0 & 0 & 0 & 0 & 0 & 0 & 0 & 1 & 0 & 1 \\
\hline Singapore & 0 & 0 & 1 & 1 & 3 & 1 & 0 & 0 & 0 & 0 & 6 \\
\hline South Africa & 0 & 0 & 0 & 0 & 0 & 0 & 0 & 1 & 0 & 0 & 1 \\
\hline Sudan & 0 & 0 & 0 & 0 & 0 & 0 & 0 & 0 & 0 & 1 & 1 \\
\hline Tanzania & 0 & 0 & 0 & 0 & 0 & 0 & 0 & 0 & 0 & 1 & 1 \\
\hline Thailand & 0 & 1 & 0 & 0 & 1 & 0 & 3 & 0 & 2 & 3 & 10 \\
\hline Vietnam & 0 & 0 & 0 & 0 & 0 & 1 & 0 & 3 & 3 & 4 & 11 \\
\hline Yemen & 0 & 0 & 1 & 0 & 0 & 0 & 0 & 0 & 0 & 0 & 1 \\
\hline Unknown & 0 & 2 & 0 & 0 & 0 & 0 & 0 & 1 & 0 & 0 & 3 \\
\hline
\end{tabular}

into 3 genotypes. All strains from 2002, 2003, and 2004 clustered in the genotype IV, and all strains from 2001, 2005, 2006, and 2008 clustered in the genotype I. Strains from 2007 and 2010 separated into both genotypes. One strain from 2009 clustered in the genotype I, whilst the other 2 strains clustered in the genotype $\mathrm{V}$.

Table 2 Serotypes and the countries of origin of the imported strains in phylogenetic trees

\begin{tabular}{lcc}
\hline Strain number & Serotype & Country of origin \\
\hline $05-226$ & 1 & Singapore \\
$05-464$ & 2 & Indonesia \\
$07-5668$ & 1 & Thailand \\
$07-5757$ & 1 & Malaysia \\
$08-7849$ & 1 & Vietnam \\
$09-1081$ & 3 & Vietnam \\
$09-9104$ & 1 & Saudi Arabia \\
$09-9236$ & 1 & Saudi Arabia \\
$09-11562$ & 1 & Thailand \\
$09-13105$ & 3 & Thailand \\
\hline
\end{tabular}

Phylogenetic analysis of DENV-2

Only 1 strain of DENV-2 was isolated in 2005, whereas 2 strains were isolated in 2010. Three E gene sequences of DENV-2 were aligned using ClustalW and analyzed with 20 reference sequences from GenBank. Figure 3 shows the phylogenetic tree of the 23 sequences with

Table 3 Serotype distributions of sequenced DENV strains in 2001-1010

\begin{tabular}{cccccc}
\hline Year & DENV-1 & DENV-2 & DENV-3 & DENV-4 & Total \\
\hline 2001 & 1 & 0 & 0 & 0 & 1 \\
2002 & 11 & 0 & 0 & 0 & 11 \\
2003 & 1 & 0 & 0 & 0 & 1 \\
2004 & 1 & 0 & 0 & 0 & 1 \\
2005 & 2 & 1 & 0 & 0 & 3 \\
2006 & 27 & 0 & 0 & 0 & 27 \\
2007 & 7 & 0 & 0 & 0 & 7 \\
2008 & 1 & 0 & 0 & 0 & 1 \\
2009 & 3 & 0 & 7 & 0 & 10 \\
2010 & 6 & 2 & 6 & 14 & 28 \\
Total & 60 & 3 & 13 & 14 & 90 \\
\hline
\end{tabular}




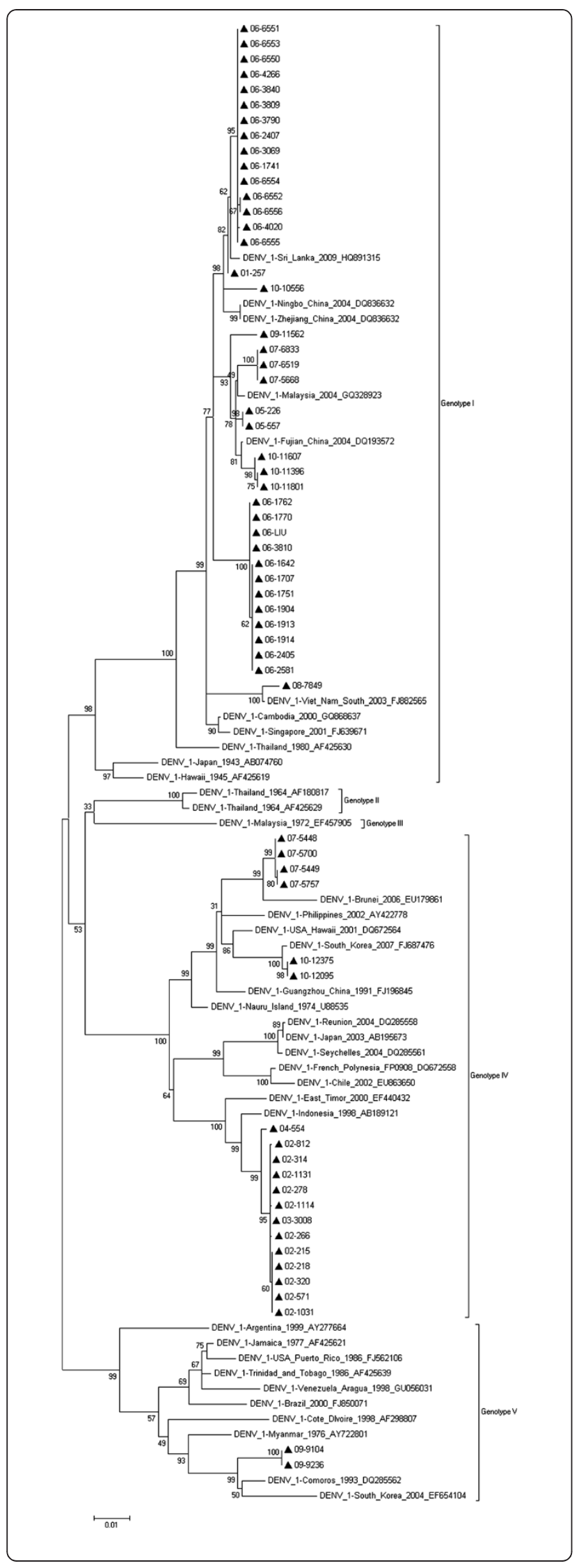

Figure 2 Phylogenetic tree of the DENV-1 E gene isolated in Guangzhou between 2001 and 2010. Sixty sequences from isolated strains and 38 reference sequences from GenBank were aligned using ClustalW. Phylogenetic trees were constructed with the maximum parsimony and maximum likelihood methods with Kimura 2-parameter corrections of multiple substitutions. Guangzhou isolates are indicated with a black triangle.

maximum likelihood analysis. All 3 DENV-2 strains were found to belong to the Cosmopolitan genotype.

\section{Phylogenetic analysis of DENV-3}

Thirteen strains of DENV-3 were isolated between 2001 and 2010, 7 in 2009, and 6 in 2010. The strains were aligned using ClustalW and analyzed with 22 reference sequences from GenBank. Figure 4 shows the phylogenetic tree of the 35 sequences with maximum likelihood analysis. The 13 DENV-3 strains consisted of genotype III (9) and genotype V (4).

\section{Phylogenetic analysis of DENV-4}

All 14 DENV-4 strains were isolated in 2010. They were aligned using ClustalW and analyzed with 11 reference sequences from GenBank. Figure 5 shows the phylogenetic tree of the 25 sequences with maximum likelihood analysis. All the 14 isolates were clustered in genotype II.

\section{Discussion}

Guangzhou has an ideal environment for DENV transmission; it has a subtropical climate, a population of over 10 million, presence of the vector mosquito, and frequent interaction with countries that have DENV epidemics. In total, over 10,000 DENV infection cases and 9 major outbreaks (over 200 infection cases each time) have been reported in the last 34 years in China $[14,16]$. The statistical data from the Chinese Center for Disease Control and Prevention showed that from 2005 to 2010, there were 2198 reported DENV infection cases in China, and 42.63\% (937 cases) of them were in Guangzhou alone, which is the highest among all cities. Given this statistic, we can say that the epidemiological and virological characteristics of DENV in Guangzhou are representative of the situation in China as a whole.

In Guangzhou, the rainy season extends from April to September, and the average temperature in Guangzhou between April and October is usually above $20^{\circ} \mathrm{C}$ [17] Our study showed that the density of Ae. albopictus and its larvae reached the highest level between June and August in Guangzhou [18]. Those facts can explain why the DENV epidemic in Guangzhou peaks between August and October.

To determine which strains were prevalent in Guangzhou, we performed phylogenetic analysis on the full-length $\mathrm{E}$ gene sequenced from 90 DENV strains that had been 


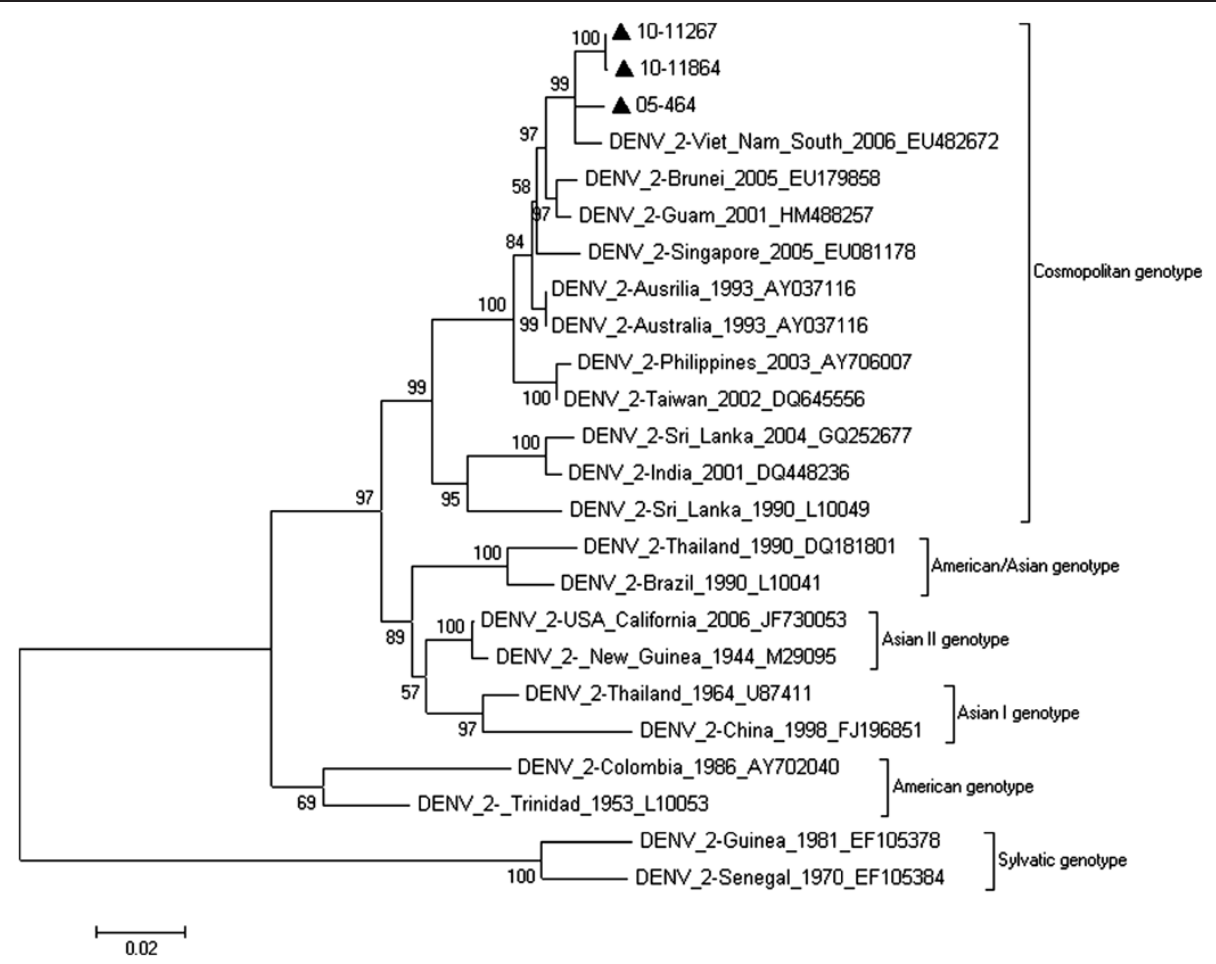

Figure 3 Phylogenetic tree of the DENV-2 E gene isolated in Guangzhou between 2001 and 2010. Three sequences from isolated strains and 20 reference sequences from GenBank were aligned using ClustalW. Phylogenetic trees were constructed with the maximum parsimony and maximum likelihood methods with Kimura 2-parameter corrections of multiple substitutions. Guangzhou isolates are indicated with a black triangle.

isolated from clinical samples [19]. DENV-1 was predominant in most of the years between 2001 and 2010 (Table 2). It was also the only serotype detected every year between 2001 and 2008, except in 2005. However, in 2009, infections caused by DENV-1, 3, and 4 serotypes occurred, and the incidence of DENV-3 prevailed over DENV-1. Then, in 2010, all 4 serotypes appeared, and again, DENV3 predominated. The phylogenetic tree of DENV-1 showed that genotype replacement occurred during this decade. The DENV-1 strain observed in 2001 was of genotype I; however, it was replaced by genotype IV in 2002, 2003 and 2004. In 2005, the dominant strain, again, was genotype I, and it remained predominant until 2010. In 2007 and 2010, both genotype I and genotype IV co-circulated. In 2009, a new genotype, genotype V occurred. Interestingly, the strains in the phylogenetic tree of DENV-2, 3, and 4 show less diversity. The DENV-2 and 4 strains belonged to only one genotype, whereas the DENV-3 strains were divided into two genotypes. There is little evidence to support the idea that there were genotype shifts in DENV-2, 3, and 4 . It could be possible that they all just appeared from 2009 (except for DENV-2 that occurred in 2005). As we have demonstrated, a lengthy longitudinal study is required to observe these shifts.

After years of investigation, we found no serotype or genotype was established in Guangzhou. Among all the
DENV infection cases reported in Guangzhou, 80 (3.23\%) cases were imported. Imported cases were seen every year between 2001 and 2010. The sensitivity of the population may also be another reason. No death or DHF/DSS case was found in the 2478 DENV infection cases during this decade. This incidence is quite low compared with other Southeast Asia countries. The incidence rate of DHF/DSS in Malaysia, Singapore, and Vietnam was 4.07-8.5, 10.47, and 51.01-77.65 per 1 million people from 2001 to 2010, as indicated on the WHO website [20]. Research has shown that some human genes are associated with DENV infection severity [21]. It could be that the people in Guangzhou are not as sensitive to DENV as the residents from other countries. Further genetic and epidemiological research would be required to determine if this is the case.

Our epidemiological investigation showed that Southeast Asian countries were the main sources (86.25\%) of the imported DENV cases (Table 2). The WHO reported that the Southeast Asian region together with the Western Pacific region bears nearly $75 \%$ of the current global dengue burden. At present, from these regions, only the Democratic People's Republic of Korea has no reports of indigenous dengue cases [22]. Southeast Asian countries are the favored travel destination for people living in Guangzhou [23]. Most of these Southeast Asian countries have had severe DENV epidemics and all 4 serotypes have, 


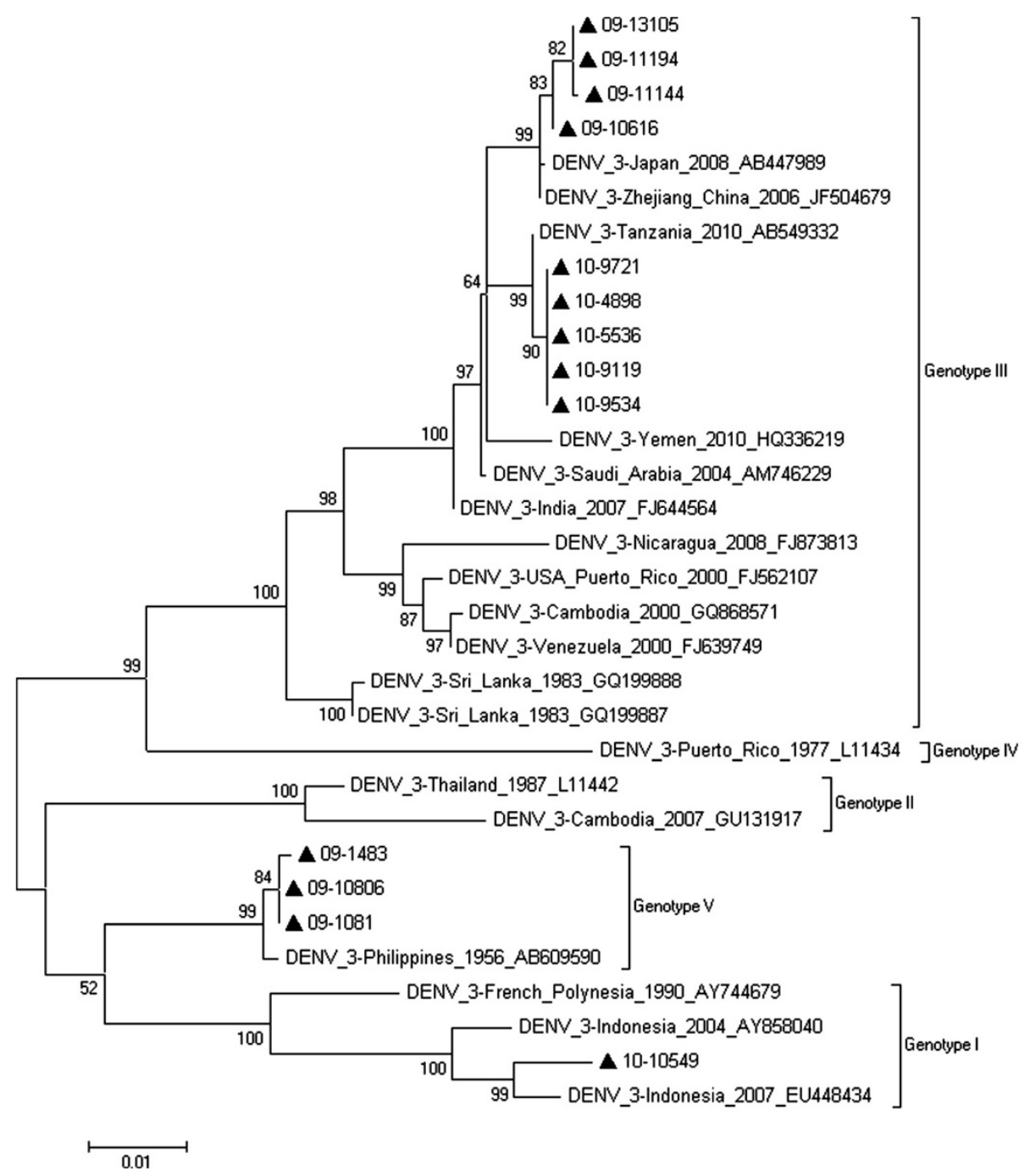

Figure 4 Phylogenetic tree of the DENV-3 E gene isolated in Guangzhou between 2001 and 2010. Thirteen sequences from isolated strains and 22 reference sequences from GenBank were aligned in ClustalW. Phylogenetic trees were constructed with the maximum parsimony and maximum likelihood methods with Kimura 2-parameter corrections of multiple substitutions. Guangzhou isolates are indicated with a black triangle.

at some point in time, been in circulation. The increased risk of DENV infection for travelers in Southeast Asia therefore increases the risk of transmission to local people in Guangzhou. The BLAST results and the phylogenetic trees of the isolated strains support this hypothesis. For the imported cases, the phylogenetic analysis verified our epidemiological data. For the local cases, most of the strains in Guangzhou were closely related to the strains isolated in prior epidemics in Southeast Asian countries. For DENV-1, the isolates in 2002 were close to the strains in Indonesia in 1998; those in 2007 were close to the strains in Malaysia in 2004; and those in 2008 were close to the strains in Vietnam in 2003. For 2010, the DENV-2 isolates were close to the strains in Vietnam in 2006, the DENV-3 isolates were close to the strains in Indonesia in
2007, and the DENV-4 isolates were close to the strains in Indonesia in 2004. A similar outcome has been reported by $\mathrm{Wu}$ et al. [24]. These results provide evidence to support the theory that Southeast Asian countries are the possible source of DENV infection in Guangzhou.

DENV-1 caused 2 major outbreaks in Guangzhou between 2001 and 2010, that is, one in 2002 (1423 cases) and another in 2006 (779 cases). Although they had the same serotype and similar impact, the strains causing the 2 outbreaks had different origins. The phylogenetic trees showed that the isolates from those two outbreaks had 2 different genotypes. The isolates in 2002 belonged to genotype IV and were close to the strain separated in Indonesia in 1998. At the beginning of the outbreak in Guangzhou in 2002 (May), a patient who came from 


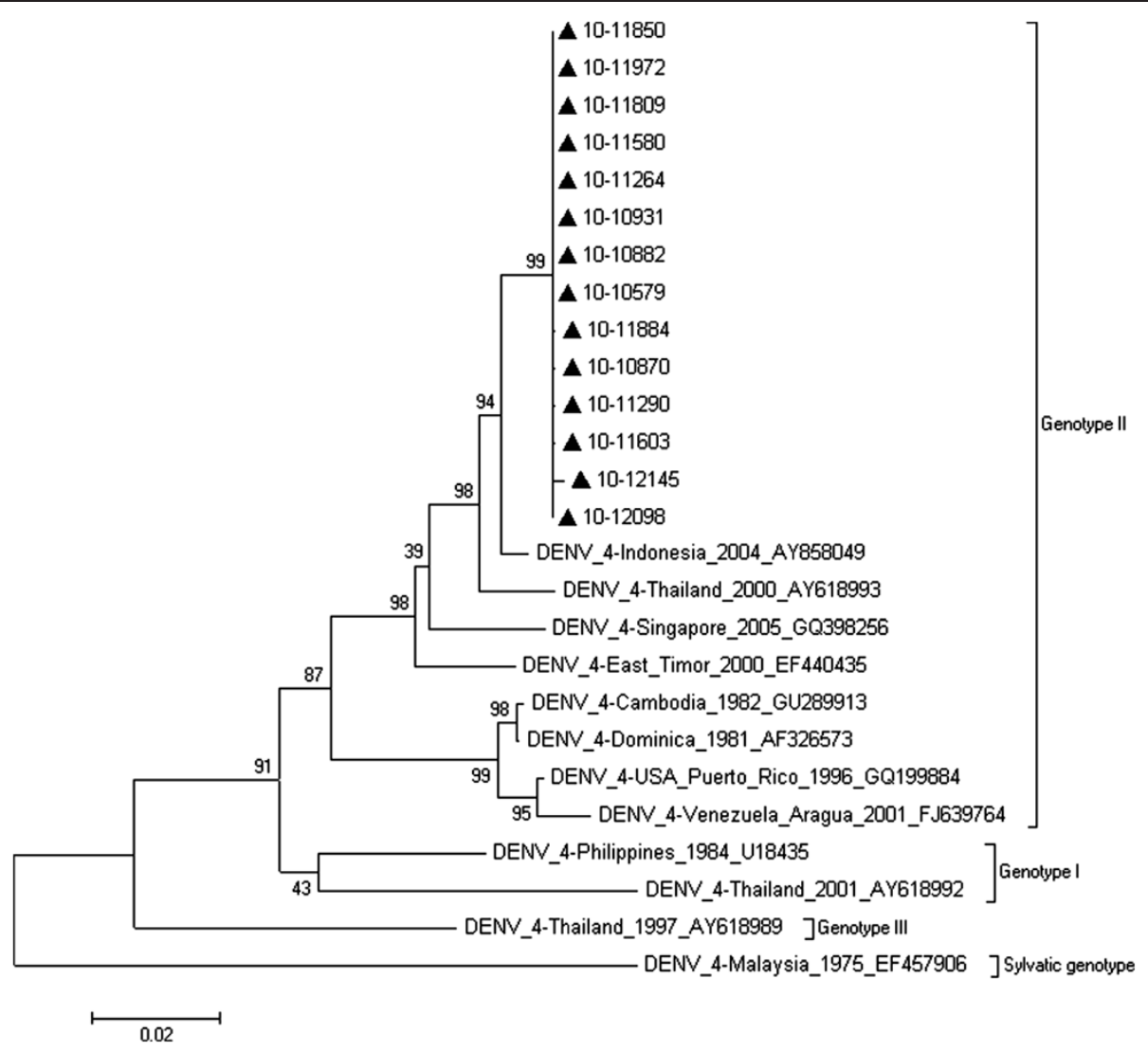

Figure 5 Phylogenetic tree of the DENV-4 E gene isolated in Guangzhou between 2001 and 2010. Fourteen sequences from isolated strains and 11 reference sequences from GenBank were aligned using ClustalW. Phylogenetic trees were constructed with the maximum parsimony and maximum likelihood methods with Kimura 2-parameter corrections of multiple substitutions. Guangzhou isolates are indicated with a black triangle.

Indonesia was found to have DENV infection. He was the first patient to be infected in 2002; however, viral isolation was unsuccessful in this case. Therefore, it cannot be concluded that the prevalent strain in 2002 came from Indonesia. Similarly, the isolates in 2006 belonged to genotype I. Cambodia was the most prevalent source of DENV infection cases in 2006. There were 8 import cases in Guangzhou that year, 4 (50\%) of which came from Cambodia. They were detected in April, August, September, and October. This indicates that in 2006, DENV infection was detected in patients coming from Cambodia at the beginning and during the peak of the outbreak. This is similar to the situation that occurred in 2002, and again, no imported strain was isolated in 2006. Therefore, because of the absence of virus strains from these imported cases, and the lack of epidemiology information in Indonesia (2002) and Cambodia (2006), it's insufficient to draw a conclusion. However, there may be some connection between the outbreaks and the imported cases.

The diversity in DENV genotypes and serotypes has increased in recent years; almost only DENV-1 was separated during 2001-2008, in contrast, the strains from 2010 included not only all 4 serotypes, but also different genotypes within the same serotype. Researchers believe that the introduction of a new serotype or genotype in an area brings with it a high possibility of a new epidemic. The high diversity of DENV in recent years indicates that there is a hidden risk of DENV epidemics occurring in Guangzhou in the near future, probably caused by the import of strains with new serotypes or genotypes.

\section{Conclusions}

Our study suggested that August to October is the epidemic season of DENV. Strain and genotype shift were frequent during the last decade and no serotype or genotype was established in Guangzhou. DENV strains from different years had different origins. Southeast Asian countries were found to be the most likely source of DENV in Guangzhou. Along with the increasing numbers of imported DENV cases and the diversity of DENV 
strains, Guangzhou is at an increased risk of DENV outbreak in the near future.

\section{Methods}

\section{Ethics statement}

This study was approved by the Ethics Committee of the Guangzhou Center for Disease Control and Prevention. Written informed consent was obtained from all participants in the study.

\section{Viruses}

The DENV strains used in this study were isolated from patients' sera, which were collected by the Guangzhou Center for Disease Control and Prevention (Guangzhou CDC) from 2001 to 2010 in Guangzhou City. The serum samples were diluted 50 fold with RPMI-1640 (Gibco, USA) and then used to inoculate a $\mathrm{C} 6 / 36$ cell monolayer, which was then incubated at $28^{\circ} \mathrm{C}$ for $10-14 \mathrm{~d}$. The DENV positive cell culture was verified by indirect immunofluorescence(IIF) test. The culture supernatant was recovered by centrifugation and stored at $-80^{\circ} \mathrm{C}$.

\section{RNA extraction, RT-PCR, and sequencing}

RNA was extracted from cell culture supernatants using QIAamp Viral RNA Mini kit (Qiagen, Germany), according to the manufacturer's instructions. cDNA was synthesized by reverse transcription with SuperScript II Reverse Transcriptase (Invitrogen, USA). The procedure followed the manufacturer's instruction. Two primers were designed to amplify the full length E gene (1.7 kb): DEN750 (5'-CAA GAACCGAAACGTGGATG-3') and DEN2639 (5'-TGTG GAAGCAAATATCACCTG-3'). The PCR was performed using $0.5 \mu \mathrm{L}$ of each primer and $4 \mu \mathrm{L}$ of cDNA, which were added to $45 \mu \mathrm{L}$ Platinum PCR SuperMix (Invitrogen, USA). The PCR reaction was initiated with a denaturation step at $94^{\circ} \mathrm{C}$ for $2 \mathrm{~min}$; followed by 40 cycles of denaturation $\left(94^{\circ} \mathrm{C}, 30 \mathrm{sec}\right)$, primer annealing $\left(52^{\circ} \mathrm{C}, 30 \mathrm{sec}\right)$ and primer extension $\left(68^{\circ} \mathrm{C}, 3 \mathrm{~min}\right)$; and ended with an extension step at $72^{\circ} \mathrm{C}$ for $7 \mathrm{~min}$. Amplified products were purified by agarose gel electrophoresis and sequenced using ABI 3730 Genetic Analyzers (Applied Biosystems, USA).

\section{Phylogenetic analysis}

Ninety sequences from isolated strains and 91 reference sequences from GenBank were aligned with ClustalW implemented in the MEGA software version 4.0 package. Phylogenetic trees were constructed with the maximum parsimony and maximum likelihood (ML) methods incorporated in the MEGA software version 4.0 package by using the Kimura 2-parameter model. The bootstrap value was 1000 replicates and only values over 50 are presented.

\section{Competing interests}

The authors declare that they have no competing interests.

\section{Authors' contributions}

$\downarrow J$ carried out most of the experiments and wrote the material and methods section. XW designed the experiments and wrote most of the manuscript. $Y W, Z B$, and $Y X$ performed virus cultures and gene sequencing. $Q J, L L$, and ZD collected the epidemiological information. YC, DB, and YW participated in DENV detection analysis. ZY and MW performed the data analysis. All authors read and approved the final manuscript.

\section{Acknowledgements}

This study was supported by the American National Institutes of Health (grant number: 1R01Al083202-01A1); the National Nature Foundation of China (grant number: 81273139 and 81271880); the Sci-tech Research Project of Guangdong Province, China (grant number: 2008B030303041); Sci-tech Research Project of Guangzhou Municipality, China (grant numbers: $2009 \mathrm{J1}$ C161 and 2011 J4300061); and Medical Sci-tech Research Projects of Guangzhou Municipality, China (grant numbers: 20121A021019, 20121A011122 and 20121A011125).

Received: 23 March 2012 Accepted: 11 December 2012 Published: 2 January 2013

\section{References}

1. Halstead S: Pathogenesis of dengue: challenges to molecular biology. Science 1988, 239:476-481.

2. Halstead S: Dengue. Lancet 2007, 370:1644-1652.

3. Kliks SC, Nimmanitya S, Nisalak A, Burke DS: Evidence that maternal dengue antibodies are important in the development of dengue hemorrhagic fever in infants. AmJTrop Med Hyg 1988, 38:411-419.

4. Kliks SC, Nisalak A, Brandt WE, Wahl L, Burke DS: Antibody-dependent enhancement of dengue virus growth in human monocytes as a risk factor for dengue hemorrhagic fever. AmJTrop Med Hyg 1989, 40:444-451.

5. WHO: Dengue/dengue haemorrhagic fever. Wkly Epidemiol Rec 2000, 75:193-196.

6. Kyle JL, Harris E: Global spread and persistence of dengue. Annu Rev Microbiol 2008, 62:71-92

7. Gubler DJ: The global emergence/resurgence of arboviral diseases as public health problems. Arch Med Res 2002, 33:330-342.

8. Wilder Smith A, Schwartz E: Dengue in travelers. N Engl J Med 2005 353:924-932.

9. Kumar SRP, Patil JA, Cecilia D: Evolution, dispersal and replacement of American genotype dengue type 2 viruses in India (1956-2005): selection pressure and molecular clock analyses. J Gen Virology 2010, 91:707-720.

10. Mendez JA, Usme-Ciro JA, Domingo C: Phylogenetic history demonstrates two different lineages of dengue type 1 virus in Colombia. Virol J 2010, 7:226-237

11. Shu P, Su C, Liao T: Molecular characterization of dengue viruses imported into Taiwan during 2003-2007: geographic distribution and genotype shift. AmJTrop Med Hyg 2009, 80:1039-1046.

12. Wittke V, Robb TE, Thu HM, Nisalak A, Nimmannitya S, Kalayanrooj S, Vaughn DW, Endy TP, Holmes EC, Aaskov JG: Extinction and rapid emergence of strains of dengue virus during an interepidemic period. Virology 2002, 301:148-156.

13. Lanciotti RS, Lewis JL, Gubler DJ, Trent DW: Molecular evolution and epidemiology of dengue-3 viruses. J Gen Virology 1994, 75:65-75.

14. Qiu F, Gubler DJ, Liu J, Chen Q: Dengue in China: a clinical review. Bull World Health Organ 1993, 71:349-359.

15. Tamura K, Dudley J, Nei M, Kumar S: MEGA4: molecular evolutionary genetics analysis (MEGA) software version 4.0. Mol Biol Evol 2007, 24:1596-1599.

16. Sun J, Lin J, Yan J, Fan W, Lu L, Huakun LV, Hou J, Ling F: Dengue virus serotype 3 subtype III,Zhejiang province,China. Emerg Infect Dis 2011, 17:321-323.

17. Bintang $Y$, Zhiying $Z$, Dezhong $X$ : Correlation between dengue fever epidemic and climate factors in Guangdong Province. J Fourth Mil Med Univ 2003, 24:143-146.

18. Zi-giang $\mathrm{Y}$, Zhi-gang $\mathrm{H}$, Yi-min J: Factors affecting the larva density index of aedes albopictus in Guangzhou. J Trop Med 2010, 5:606-608.

19. Rico-Hesse R: Microevolution and virulence of dengue viruses. Adv Virus Res 2003, 59:315-341. 
20. WHO: Dengue net database. http://apps.who.int/globalatlas/DataQuery/ defaultasp.

21. Stephens HA: HLA and other gene associations with dengue disease severity. Curr Top Microbiol Immunol 2010, 338:99-114.

22. WHO: Situation update of dengue in the SEA region:; 2010. http://209.61.208.233/LinkFiles/Dengue_Dengue_update_SEA_2010.pdf.

23. Jian $D$, Lin-fang L: A study on tourist behavior of outbound travel for Guangzhou resident. Geogr Res 2004, 23:705-714.

24. Wu W, Bai Z, Zhou H, Tu Z, Fang M, Tang B, Liu J, Liu L, Liu J, Chen W: Molecular epidemiology of dengue viruses in southern China from 1978 to 2006. Virol J 2011, 8:322-330.

doi:10.1186/1743-422X-10-4

Cite this article as: Jiang et al:: Molecular epidemiological and virological study of dengue virus infections in Guangzhou, China, during 2001-2010. Virology Journal 2013 10:4.

\section{Submit your next manuscript to BioMed Central and take full advantage of:}

- Convenient online submission

- Thorough peer review

- No space constraints or color figure charges

- Immediate publication on acceptance

- Inclusion in PubMed, CAS, Scopus and Google Scholar

- Research which is freely available for redistribution 